ARTICLE OPEN

\title{
Proteasome-targeted nanobodies alleviate pathology and functional decline in an $\alpha$-synuclein-based Parkinson's disease model
}

\author{
Diptaman Chatterjee ${ }^{1}$, Mansi Bhatt ${ }^{1}$, David Butler ${ }^{2,3}$, Erwin De Genst ${ }^{4}$, Christopher M. Dobson ${ }^{4}$, Anne Messer ${ }^{2,3}$ and $^{2}$ \\ Jeffrey H. Kordower ${ }^{1,5}$
}

Therapeutics designed to target a-synuclein (a-syn) aggregation may be critical in halting the progression of pathology in Parkinson's disease (PD) patients. Nanobodies are single-domain antibody fragments that bind with antibody specificity, but allow readier genetic engineering and delivery. When expressed intracellularly as intrabodies, anti-a-syn nanobodies fused to a proteasome-targeting proline, aspartate or glutamate, serine, and threonine (PEST) motif can modulate monomeric concentrations of target proteins. Here we aimed to validate and compare the in vivo therapeutic potential of gene therapy delivery of two proteasome-directed nanobodies selectively targeting a-syn in a synuclein overexpression-based PD model: VH14*PEST (nonamyloid component region) and NbSyn87*PEST (C-terminal region). Stereotaxic injections of adeno-associated viral 5-a-syn (AAV5a-syn) into the substantia nigra (SN) were performed in Sprague-Dawley rats that were sorted into three cohorts based on preoperative behavioral testing. Rats were treated with unilateral SN injections of vectors for VH14*PEST, NbSyn87*PEST, or injected with saline 3 weeks post lesion. Post-mortem assessments of the SN showed that both nanobodies markedly reduced the level of phosphorylated Serine-129 a-syn labeling relative to saline-treated animals. VH14*PEST showed considerable maintenance of striatal dopaminergic tone in comparison to saline-treated and NbSyn87*PEST-treated animals as measured by tyrosine hydroxylase immunoreactivity (optical density), DAT immunoreactivity (optical density), and dopamine concentration (high-performance liquid chromatography). Microglial accumulation and inflammatory response, assessed by stereological counts of Iba-1-labeled cells, was modestly increased in NbSyn87*PEST-injected rats but not in VH14*PEST-treated or saline-treated animals. Modest behavioral rescue was also observed, although there was pronounced variability among individual animals. These data validate in vivo therapeutic efficacy of vector-delivered intracellular nanobodies targeting a-syn misfolding and aggregation in synucleinopathies such as PD.

npjParkinson's Disease (2018)4:25 ; doi:10.1038/s41531-018-0062-4

\section{INTRODUCTION}

Synucleinopathies are a class of neurodegenerative diseases featuring misfolding and disordered aggregation of the protein, a-synuclein (a-syn). Parkinson's disease (PD) is the most common of these disorders and is primarily characterized by significant loss of dopaminergic neurons in the substantia nigra (SN) and abrogation of dopaminergic tone along the nigrostriatal pathway. ${ }^{1,2}$ A prominent neuropathological hallmark of PD is the presence of a-syn centric intracellular inclusions throughout the brain, called Lewy bodies and Lewy neurites.,4 Although the precise mechanisms of a-syn-mediated neurotoxicity are still unknown, findings suggest that overexpression of a-syn in concert with encumbered chaperone activity and dysfunctional protein degradation machinery may trigger aggregation and cellular disruption. $^{5-9}$ Furthermore, mounting evidence of direct cell-tocell transmission of toxic a-syn species lends weight to the theory of prion-like spatiotemporal progression of pathology. ${ }^{10-12}$ Thus, methods to interrupt proteinaceous inclusion formation and proteopathic seeding represent promising paradigms for therapeutic interventions.

Currently, efforts to mediate a-syn toxicity have primarily utilized a-syn-targeting immunoglobulins to neutralize extracellular transmission of propagating species. ${ }^{13-16}$ However, uncovering methods to probe and impede intracellular induction of proteinopathy in the PD brain is a significant challenge for longterm, lasting remediation of a-syn toxicity. Intrabodies, either in single-chain variable fragment form or single-domain immunoglobulin fragments $\left(\mathrm{V}_{\mathrm{H}}, \mathrm{V}_{\mathrm{HH}}\right.$, or $\left.\mathrm{V}_{\mathrm{L}}\right)$, provide a novel therapeutic approach for intracellular targeting of disordered antigens in neurological disease. ${ }^{17}$ Numerous candidate intrabodies have been selected and tested in vitro targeting various species of asyn, including monomeric, oligomeric and protofibrillar, and fibrillar forms (reviewed by: Bhatt et al. ${ }^{18}$ and De Genst et al. ${ }^{19}$ ). Previously we have characterized in situ efficacy of two primary aptamer nanobodies, $\mathrm{VH} 14$ (otherwise referred to as NAC14) ${ }^{20}$ and NbSyn $87,{ }^{21}$ to interfere with mutant a-syn aggregation events and dampen model system proteostatic burden. ${ }^{22}$

\footnotetext{
${ }^{1}$ Department of Neurological Sciences, Rush University Medical Center, Chicago, IL c60612, USA; ${ }^{2}$ Neural Stem Cell Institute, Regenerative Research Foundation, Rensselaer, NY 12144, USA; ${ }^{3}$ Department of Biomedical Sciences, University at Albany, Albany, NY 12208, USA; ${ }^{4}$ Centre for Misfolding Diseases, Department of Chemistry, University of Cambridge, Cambridge CB2 1EW, UK and ${ }^{5}$ Van Andel Research Institute, Grand Rapids, MI 49503, USA Correspondence: Jeffrey H. Kordower (jkordowe@rush.edu)
} 
A.

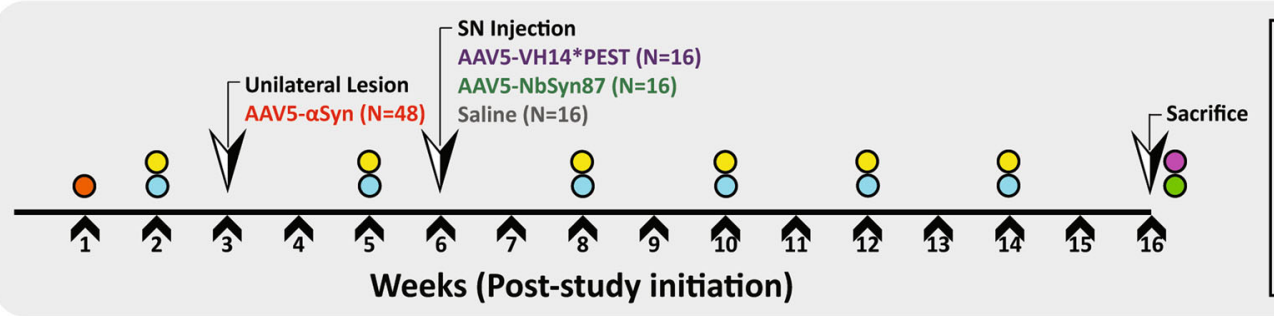

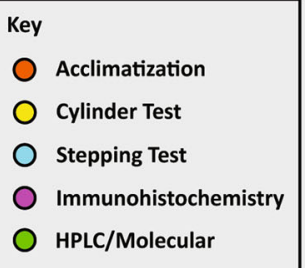

Key

Acclimatization

Cylinder Test

Stepping Test

HPLC/Molecular

B.

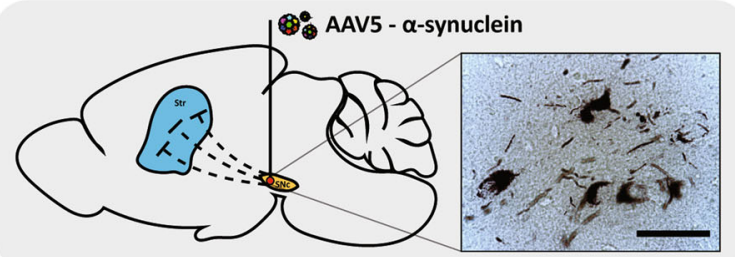

C.

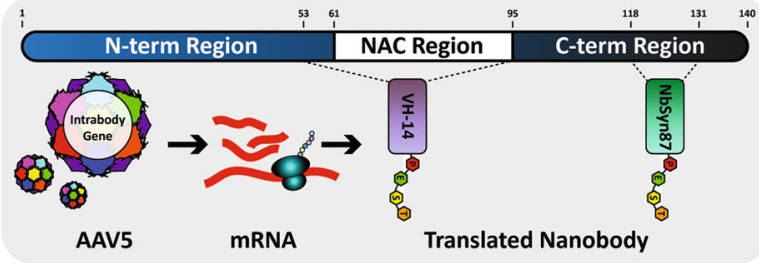

D.

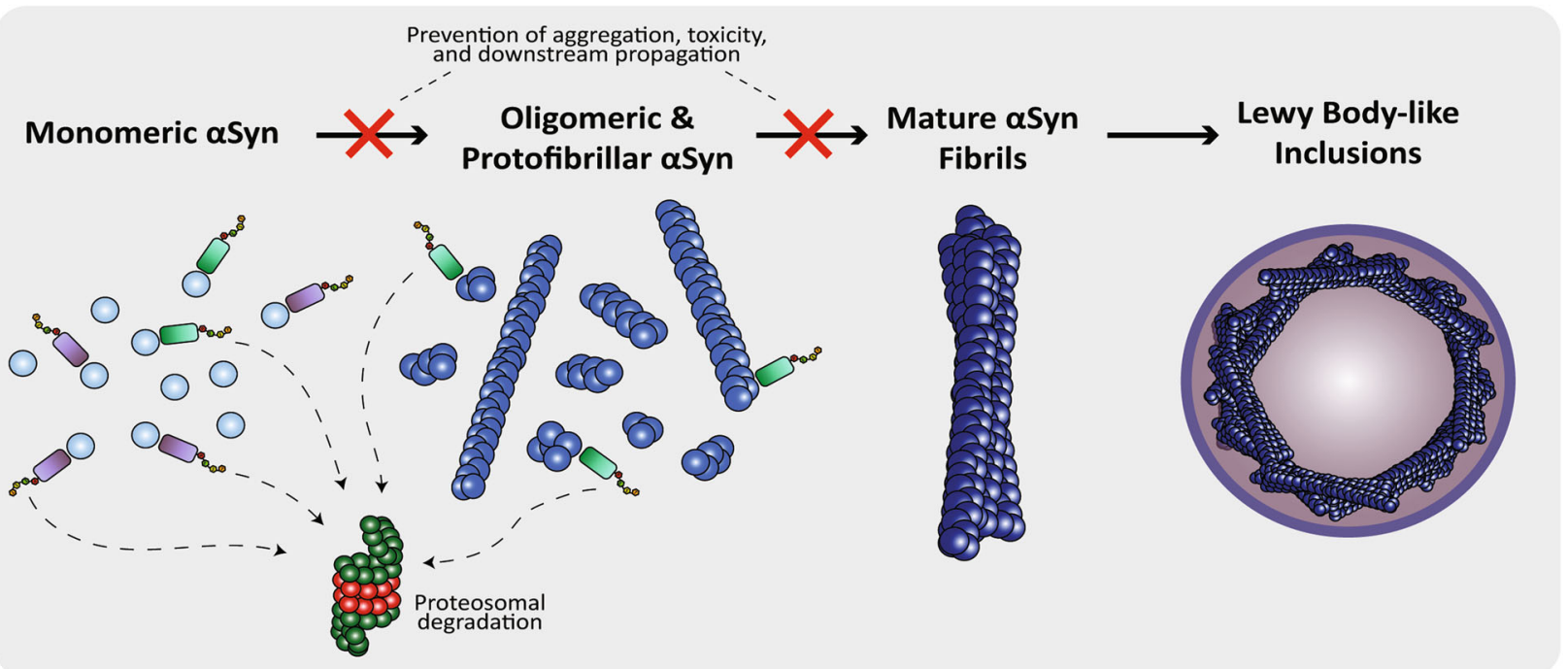

Fig. 1 Experimental design and study overview. a Study design: genes expressing intrabodies or saline vehicle were delivered by viral vector into animals previously lesioned with an $\alpha$-syn overexpression vector for observation of treatment effects on behavior and post-mortem analysis of pathology. b Model overview of $\alpha$-syn overexpression via viral vector delivery of human $\alpha$-syn into the substantia nigra pars

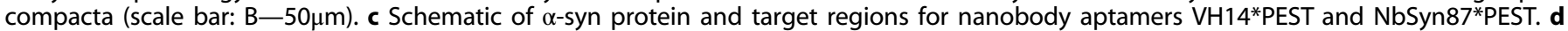
Diagram of the process of $\alpha$-syn fibril formation, sites of intrabody binding, and intrabody-mediated protein clearance mechanisms

VH14 was screened from a public access, yeast surface display library and has a high-binding affinity for the hydrophobic nonamyloid component region of monomeric a-syn (Fig. 1a, c) found to be critical in fibril formation. ${ }^{20} \mathrm{VH} 14$ is a nanobody derived from human origin, making it a prime candidate for evading immunogenicity and inflammatory perturbation in the context of clinical PD. NbSyn87 is a fully camelid nanobody selected from a phage display library. ${ }^{23} \mathrm{NbSyn} 87$ belongs to a class of nanobody aptamers that target the C-terminal region of a-syn (Fig. 1c, d) that is known to be the site of post-translational modifications affecting pathogenic triggers of misfolding. ${ }^{24}$ As the C-terminal tail of a-syn remains exposed in oligomeric and protofibrillar species of aggregated complexes, NbSyn87 provides the added benefit of binding to both monomeric and early fibrillar forms of a-syn, although epitope affinity is markedly reduced in advanced maturation stages. ${ }^{21}$ Moreover, NbSyn 87 and another nanobody, NbSyn2, which are both specific for the C-terminal region of a-syn, have been shown to inhibit the formation of toxic oligomers in vitro and protect against the toxic action of these oligomers in cell culture models. ${ }^{25}$

Nanobody aptamers independent of stabilizing light-chain fragments are often poorly soluble and prone to aggregation, potentially exacerbating the proteostatic burden in the context of the PD neuraxis. Previously, we have validated enhanced nanobody solubility by engineering a polypeptide tether construct rich in proline, aspartate or glutamate, serine, and threonine (PEST) residues. $^{22,26,27}$ The fusion of the PEST motif decreases the overall net charge and isoelectric point of the protein nanobody improving soluble aptamer expression and effective concentrations in vitro. PEST constructs also act as ubiquitin-independent proteosomal-targeting motifs capable of rapidly degrading bound antigen. We have previously confirmed that the fusion of a PEST degron, derived from a murine ornithine decarboxylase protein, to both VH14 and NbSyn87 efficaciously stalls a-syn aggregation, mediates proteasomal clearance of a-syn, and attenuates proteopathic stress. $^{22}$

Here, we demonstrate in vivo, proof-of-concept efficacy data of two candidate intrabodies, VH14*PEST and NbSyn87*PEST, delivered by gene therapy to a viral a-syn overexpression rodent model of PD. This study validates the proposition that both engineered nanobodies can reduce aggregation of a-syn and limit the expression of $a$-syn species phosphorylated at Serine-129 ( $p$ S129) correlative of pathological aggregation. Additionally, our data suggest that VH14*PEST a-syn targeting attenuates functional indices reflective of nigrostriatal dopaminergic integrity. These data corresponded with modest preservation in gross 
motor behavior in the VH14*PEST-treated cohort. Although displaying inferior rescue of striatal dopaminergic output measures in comparison with VH14*PEST, NbSyn87*PEST treatment also showed indications of protected motor function. Collectively, these data provide a platform for further pre-clinical screening of a-syn targeting intrabodies as potential therapeutics for PD and other synucleinopathies.

\section{RESULTS}

VH14*PEST and NbSyn87*PEST expression reduces pathologic aggregation of $a-s y n$

To model a-syn-based proteinopathy, we utilized a viral vector overexpression model of human wild-type a-syn. All subjects received a unilateral injection of an AAV5-a-syn construct directly into the substantia nigra (Fig. 1b). Contralateral hemispheres were not injected and used as controls for lesioned hemispheres. To equilibrate baseline functional performance, lesioned animals were pre-sorted into treatment groups based on baseline behavioral function (Fig. 4). Untreated lesions generated consistent, unilateral Lewy body-like inclusions (Fig. 1b) in the soma and neurites of injected nigral neurons. These protein bodies were resistant to proteinase $\mathrm{K}$ digestion, indicating insoluble a-syn inclusions representative of pathologic aggregates (Fig. S2). Three weeks post lesion, animals were injected with viral vectors expressing $\mathrm{VH} 14^{*} \mathrm{PEST}$ or NbSyn87*PEST, or were injected with an equivalent volume of control saline. Histological analysis of post-mortem tissue revealed significant $(p<0.05)$ reduction in labeling of $\mathrm{p}-\mathrm{S} 129$, as measured densitometrically relative to the unlesioned side (Fig. 2a, b). VH14*PEST and NbSyn87*PEST treatment produces a near twofold reduction of relative pathological aggregate labeling compared to saline treatment of lesions (Fig. 2b). Inclusion formation is markedly reduced in both nanobody-treated groups, with $\mathrm{VH} 14^{*} \mathrm{PEST}$ displaying robust reduction of $\mathrm{p}-\mathrm{S} 129$ labeling (Fig. $2 \mathrm{a}$, inset). Dual immunofluorescent labeling of p-S129 a-syn and Thioflavin-S validates the labeling of $\mathrm{p}$-S129 residues corresponding with $\beta$-sheet aggregation, as is the case in authentic Lewy body pathology (Fig. 2c).

Due to some contralateral background staining observed in $\mathrm{p}$ S129 staining in the SN, we stained for human-specific a-syn species (LB509) to confirm the expression of vector-derived a-syn on the side of lesioning (Fig. S1a). Robust expression of a-syn was predominantly localized ipsilateral to the site of lesion (Fig. S1a). Densitometric measurement of LB509-labeled a-syn in the SN did not reveal statistically significant changes in treatment groups, although there were slight non-significant decreases for both VH14*PEST-treated and NbSyn87*PEST-treated cohorts (Fig. S1b). Taken together, these data demonstrate that both intrabodies reduce a-syn protein concentration at a permissive rate for inhibited proteopathic aggregation but do not excessively clear asyn species to the point of phenotypic loss of function.

VH14*PEST halts a-syn-induced pathology afflicting nigrostriatal dopaminergic tone

Given the success of both intrabodies in governing a-syn aggregation, we next aimed to evaluate nigrostriatal pathology central to canonical PD phenotype. Histological analysis of tyrosine hydroxylase (TH) labeling in the striatum revealed significant preservation of synaptic innervation in VH14*PESTtreated animals compared to both saline-treated and NbSyn $87^{*}$ PEST-treated animals (Fig. 3a). $\mathrm{TH}^{+}$deficiency of the lesioned hemispheres was more prominent in the dorsolateral region of the striatum (Fig. 3a). By contrast, relative quantitation of VH14*PESTtreated animals showed a $49 \%$ increase in striatal $\mathrm{TH}^{+}$labeling compared to saline-treated animals and a $38 \%$ increase compared to NbSyn87*PEST-treated animals. Histological analysis of $\mathrm{TH}$ labeling in the striatum revealed significant preservation of synaptic innervation in VH14*PEST-treated animals compared to both saline-treated and NbSyn $87^{*}$ PEST-treated animals.

Next, we evaluated the stereological density of $\mathrm{TH}^{+}$-labeled cells in the SN (Fig. 3a). In our hands, 4-month post-model induction was insufficient time to see significant loss in nigral dopaminergic neurons in lesioned hemispheres, as has been previously reported. ${ }^{28}$ However, intrabody treatment shows a trend in neuroprotection by one-way analysis of variance (ANOVA) comparison of $p$ values between lesioned and contralateral control nigral neuron densities (Fig. 3c). To establish a more complete profile of striatal dopaminergic capacity, we stained sections for dopamine transporter (DAT) expression (Fig. 3b). Consistent with the TH data, the VH14*PEST-treated cohorts showed modest protection of overall DAT expression with a $28 \%$ increase in optical density relative to control expression (Fig. 3c). NbSyn 87 treatment also ameliorated both TH and DAT expression, but at statistically insignificant levels (Fig. $3 a-c)$. To determine the overall supply of striatal dopamine, tissue of eight animals from each cohort was processed by high-performance liquid chromatography to determine relative dopamine concentrations. Although changes in striatal dopamine supply were statistically insignificant, median dopamine concentration in VH14*PESTtreated animals relative to the control hemispheres was $\sim 3$-fold higher than that of the saline-treated group ( $p=0.13$ ) (Fig. 3c). Individual subjects had dynamic variability in the total dopamine concentrations recorded.

To appraise intrabody-mediated changes to nigrostriatal output, we evaluated motor phenotype via two behavioral assays throughout the study: the stepping test and the cylinder test. VH14*PEST-treated animals showed significant $(p<0.05)$ improvement in motor behavior compared to saline-treated control in stepping test performance at the study endpoint (Fig. $4 a$, b). In contrast to histopathological evaluation of nigrostriatal tone, NbSyn87*PEST-treated animals showed improved rescue of the motor phenotype. NbSyn87*PEST-treated animals displayed significant increases in stepping test contralateral forelimb contacts in comparison to lesioned saline controls from 2-week post lesion through to the study endpoint (Fig. 4a, b). NbSyn87*PEST-treated animals showed less rapid preservation of motor behavior in the cylinder test performance compared to saline controls, only displaying significant improvement 6 weeks post lesion (Fig. 4c, d). In both behavioral assays, no statistically significant differences were found in motor performance between both intrabodytreated cohorts. Individual animal performance in both intrabody cohorts was highly variable, coinciding with the pronounced variance in striatal dopamine levels (Fig. 4b, d). Collectively, these data show VH14*PEST treatment to elicit significant preservation of multiple indices of nigrostriatal health and pathway maintenance in the context of induced synucleinopathy. However, the NbSyn87*PEST-treated group, although showing minimal evidence of dopaminergic rescue, only modestly conserved overall motor function.

NbSyn87*PEST elicits inflammatory response in lesioned SN

A critical aspect of gene therapy and immunoglobulin-derived treatment constructs is immunogenicity and a potentiated inflammatory response to treatment. To evaluate potential inflammation, we investigated the recruitment and activity of microglia to sites of injection in the SN. We immunolabeled the microglial calcium-binding protein marker, Iba-1, as a reporter of resident macrophage activation (Fig. 5). In comparison to unlesioned contralateral hemispheres, treated hemispheres of both the saline and VH14*PEST cohorts showed statistically insignificant increases in total microglial density, as measured stereologically (22 and 13\%, respectively) (Fig. 5b). However, NbSyn $87^{*}$ PEST treatment induced a $63 \%$ increase in microglial density compared to controls (Fig. 5b). Consistent with these 

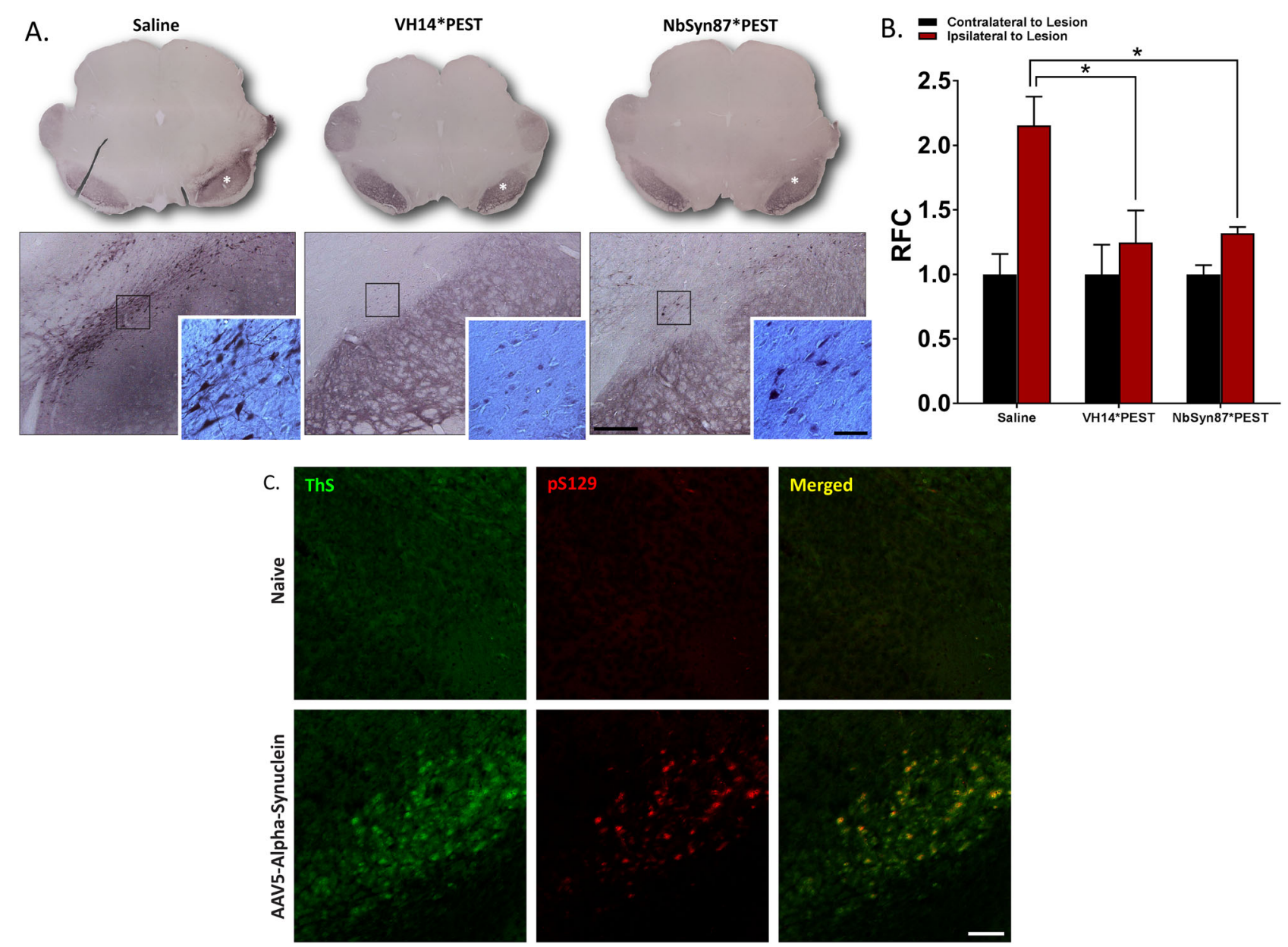

Fig. 2 Nanobody expression significantly reduces induced pathological synucleinopathy. a Labeling of phosphorylated Serine-129 $\alpha$-syn species in the substantia nigra reveals reduction of pathological aggregates in both intrabody-treated cohorts compared to saline controls. Intrabody treatment also reduces the overall level of mature Lewy body-like cellular aggregates (inset) (scale bars: 100 and $25 \mu \mathrm{m}$ ). b Quantification of optical density measurements of phospho-Serine-129 labeling in the substantia nigra. Both VH14*PEST and NbSyn87*PEST treatment groups reveal significant decreases in densitometric labeling of pathological $\alpha$-synuclein species. The data were analyzed via oneway ANOVA with Tukey's post hoc comparisons (saline- $n=8$, VH14*PEST $-n=7$, NbSyn87*PEST $-n=7$, all ${ }^{*} p<0.05$ ). c Dual immunofluorescent labeling of phosphorylated Serine-129 and Thioflavin-S confirms that phosphorylated residues are representative of aggregated $\alpha$-syn species (scale bar: $100 \mu \mathrm{m}$ )

findings, microglia in the SN of NbSyn87*PEST-treated animals appear amoeboid in morphology, generally indicative of phagocytic macrophage activity (Fig. 5a, inset, red arrows). By contrast, observations of microglial morphology for saline-treated and VH14*PEST-treated animals preserve the canonical ramified, inactive shape (Fig. 5a, inset). These assessments implicate NbSyn $87^{*}$ PEST as a potential inducer of inflammatory response, although further investigation of glial surface markers and signal transduction is necessary to reveal a true immune response.

\section{DISCUSSION}

Currently, there are no disease-modifying clinical therapies being used directly to combat the synucleinopathy central to PD pathogenesis. Numerous recent efforts have aimed to halt pathogenic cellular propagation of fibrillar species through immunotherapy with promising success. ${ }^{13-16,29}$ However, immunotherapeutic modalities fail to adequately address the intracellular pathogenic cascade. Improper a-syn nucleation and oligomerization at axon terminals are central to mechanistic implications of PD pathology. ${ }^{30}$ Clearance of extracellular a-syn deposits may not actively prevent stochastic intracellular seeding and degenerative pathway activation. ${ }^{31,32}$ Furthermore, active pathways employed by therapeutic biologics for a-syn clearance may be functionally perturbed in PD cellular environments. ${ }^{33-35}$ Thus, the prevention of intracellular aggregation and maintenance of intracellular concentrations is critical in reversal of neurotoxicity.

We have previously shown that both $\mathrm{VH} 14^{*}$ PEST and NbSyn $87^{*}$ PEST were effective in reducing excess $a$-syn accumulation and limiting a-syn-mediated neurotoxicity in vitro. ${ }^{22}$ Proteins containing a PEST motif are often targeted for proteasomal degradation. Fusion of the PEST degron to anti-huntingtin intrabodies targets them and their intracellular cargo to the proteasome for degradation based on proteasome inhibition with specific proteasome inhibitor epoxomicin. ${ }^{27}$ Additionally, scrambling the PEST motif successfully eliminates the clearance of both the intrabody and its intracellular cargo. ${ }^{27}$ These findings have also been replicated with the VH14*PEST and NbSyn87*PEST constructs used in this study. ${ }^{22}$ For these targets to be degraded through the proteasome, they must remain soluble, ${ }^{36}$ which intrabody binding facilitates. When binding to the proteasome the cargo is degraded along with the short $(<200$ amino acids) nanobody. Although it is difficult to resolve the relative contributions of intrabody interference of target epitopes or proteasomal substrate clearance in the observed antiamyloidogenesis effect of this study, our in vitro data leads us 

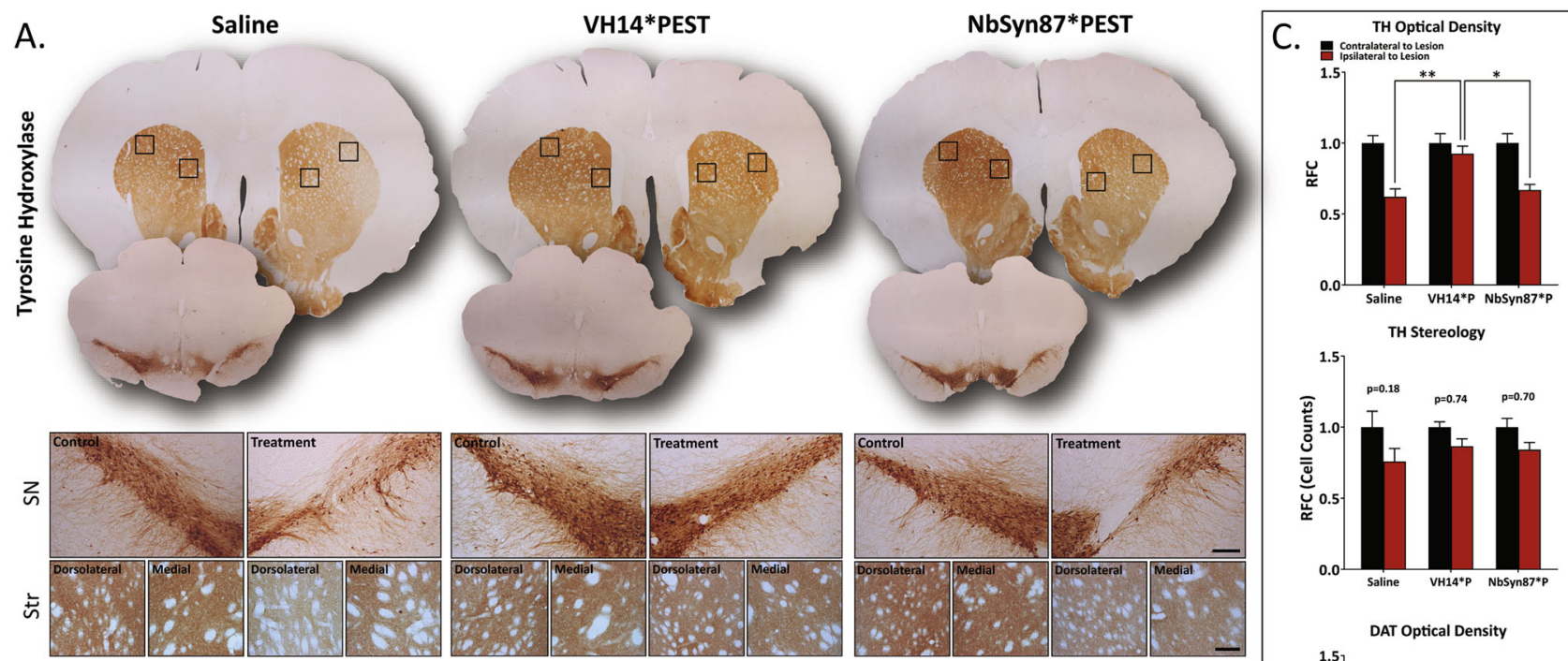

B.

Saline

VH14*PEST

\section{NbSyn87*PEST}
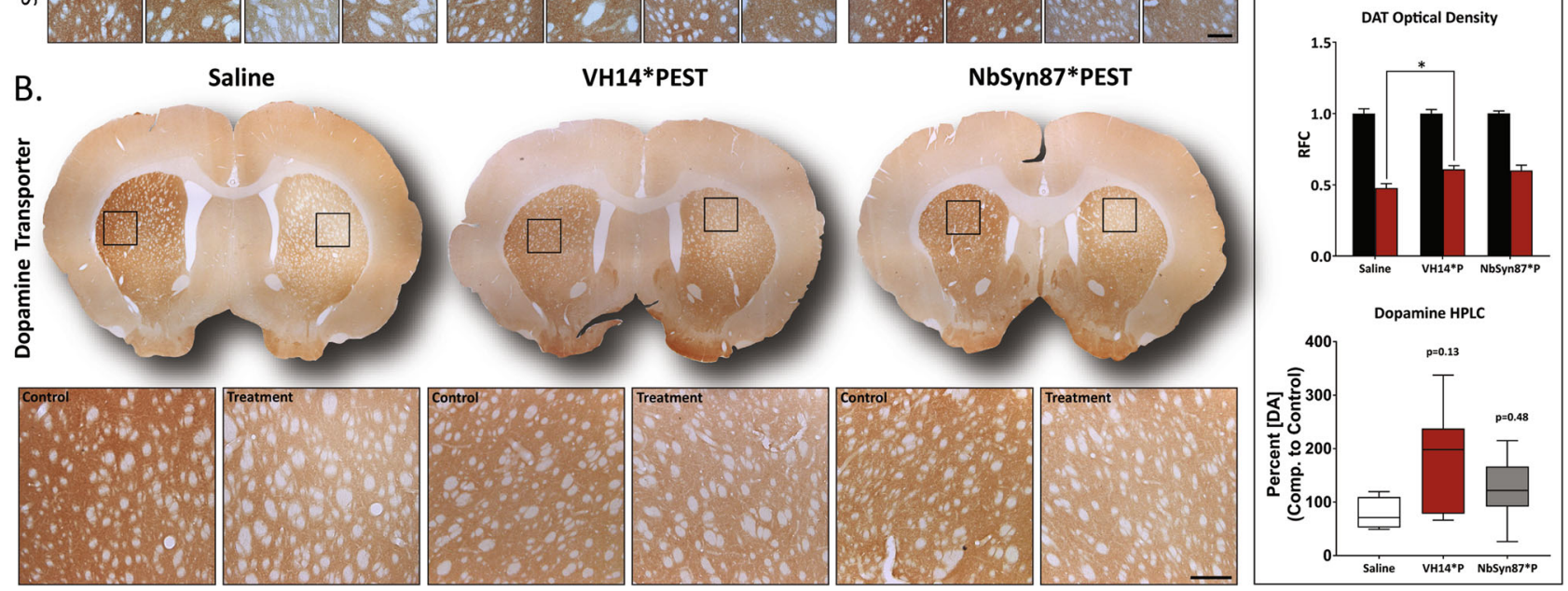

Fig. 3 VH14*PEST treatment counteracts insults in nigrostriatal dopaminergic tone induced by synucleinopathy. a Assessment of TH ${ }^{+}$ immunoreactivity in the substantia nigra and striatum. VH14*PEST reveals preservation of striatal innervation in comparison to saline-treated and NbSyn87*PEST-treated animals. Evidence of protection is strongest in the dorsolateral striatum (scale bars: 100 and $25 \mu \mathrm{m}$ ). b Immunostaining of dopamine transporter (DAT) expression in the striatum shows modest improvement in VH14*PEST treatment animals compared to saline-treated animals (scale bar: $25 \mu \mathrm{m})$. c Quantification of striatal TH optical density $\left(\right.$ saline- $n=7, \mathrm{VH} 14^{*} \mathrm{PEST}-n=7$, NbSyn $87^{*} \mathrm{PEST}-n=8$ ), stereological cell counts of TH-labeled cells in the substantia nigra (saline $-n=7, \mathrm{VH} 14^{*} \mathrm{PEST}-n=8, \mathrm{NbSyn} 87^{*} \mathrm{PEST}$ $-n=7$ ), striatal DAT optical density (saline $-n=7, \mathrm{VH} 14^{*} \mathrm{PEST}-n=8, \mathrm{NbSyn} 87^{*} \mathrm{PEST}-n=7$ ), and the concentration of striatal dopamine

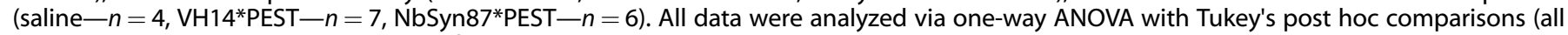
${ }^{*} p<0.05,{ }^{* *} p<0.01$, or as indicated on figure)

to hypothesize PEST-mediated a-syn clearance to be an essential factor in disaggregation.

Addition of the PEST degron to both nanobody aptamers enhanced hydrophilicity and decreased overall molecular charge to stabilize soluble constructs, allowing for superior a-syn targeting and proteosomal clearance. ${ }^{26}$ Consistent with these data are our findings of intrabody-mediated a-syn clearance and diminution of pathologic a-syn labeling in the SN (Fig. 2a, b). With Thioflavin-S staining of $\beta$-sheet aggregation, we confirmed $p$ S129-labeled species to be indicative of toxic aggregates and a representative marker for histopathology (Fig. 2c). Due to limited tissue material we were unable to appropriately quantify reductions in Thioflavin-S staining by stereology, although we were able to qualitatively observe reductions in aggregation in both intrabody-treated cohorts. We determined in previous in situ characterizations of these two candidate intrabodies that although NbSyn87*PEST clears a-syn more effectively than VH14*PEST, VH14*PEST modifies cellular stress associated with multi-factorial proteostasis to a greater extent, particularly in the case of a-syn promotion of mutant huntingtin proteinopathy. ${ }^{22}$ This aspect of intervention is integral for synucleinopathies that are commonly associated with concomitant pathology of additional proteins vulnerable to disordering or misfolding such as tau, amyloid- $\beta$, and mutant huntingtin. Abating proteostatic stress in PD, where both chaperone-mediated autophagic and macro-autophagic systems are perturbed and changes in cellular oxidation accelerate misfolding, may be a complementary clinical target to a-syn toxicity. $5,30,33,34$

This study aimed to use intrabody treatment to modify synucleinopathy in the context of canonical Parkinsonian motor pathology. Nigrostriatal pathology in PD is a complex process that is difficult to accurately and temporally model. Clinical PD begins as an axonopathy in which striatal terminal depletion and subsequent neurotransmittive failure provokes phenotypic onset. $^{37-39}$ One of the a-syn's primary roles in striatal axonopathy is phenotypic insult through synaptic injury. ${ }^{37-41}$ To that end, our model represents early PD-like pathology in which striatal innervation loss can clearly be observed through reductions in TH and DAT immunoreactive densities and depleted levels of striatal dopamine (Fig. 3). These deficits were directly correlated to a-syn aggregation that featured classical $\beta$-sheet amyloid organization (Thioflavin-S) and was resistant to Proteinase $\mathrm{K}$ 
A.

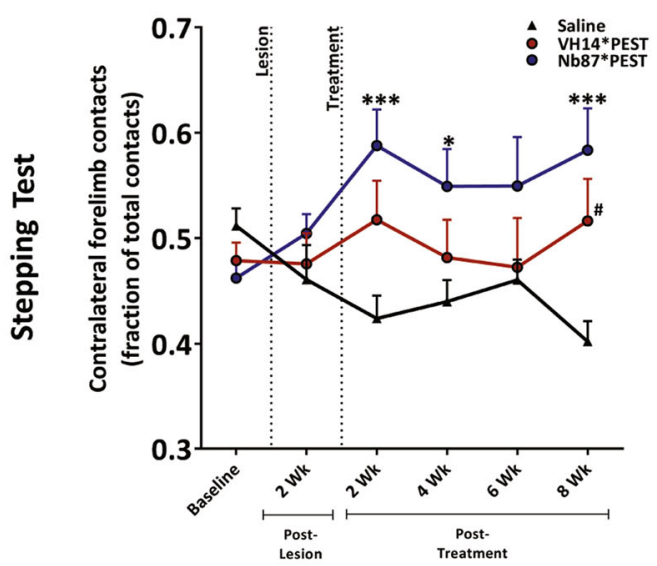

C.

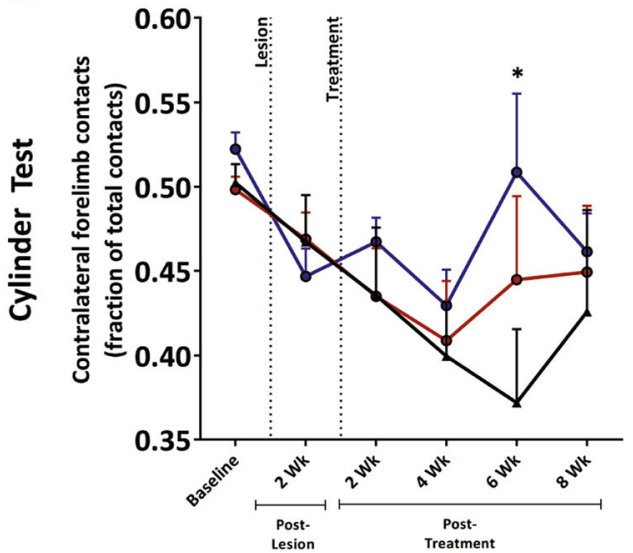

B.

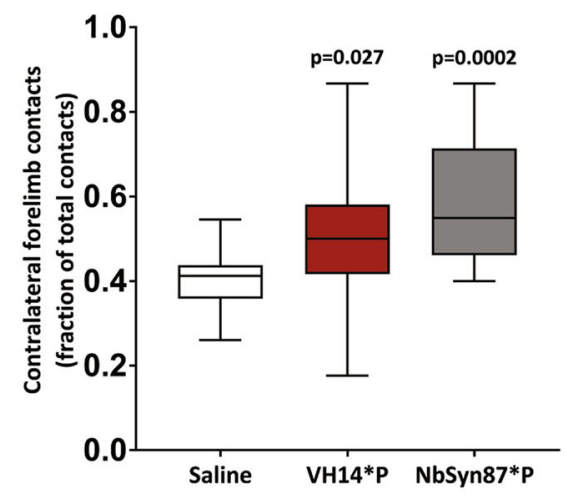

D.

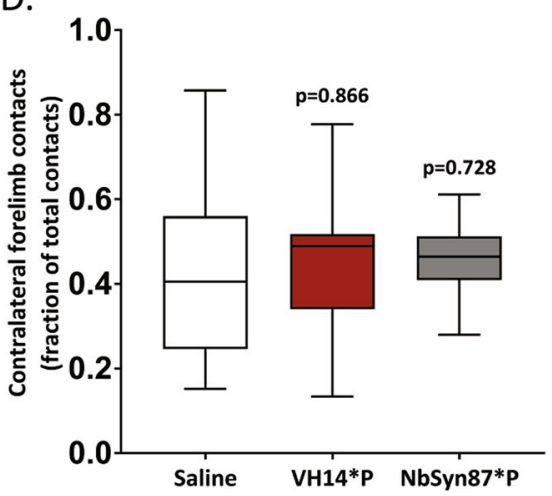

Fig. 4 Nanobody treatment induces functional motor recovery. a Results of stepping test behavior to assess unilateral forelimb akinesia induced by synucleinopathy and intrabody treatment effects. Both intrabody-treated cohorts improve use of forelimbs contralateral to site of lesion. b Box-and-whisker graph of study-endpoint stepping test behavioral performance. c Results of cylinder test behavior to assess unilateral forelimb akinesia and intrabody treatment effects. d Box-and-whisker plot of study-endpoint cylinder test behavioral performance. All the data were analyzed via two-way ANOVA with Tukey's post hoc comparisons (saline $-n=14, \mathrm{VH} 14^{*} \mathrm{PEST}-n=15, \mathrm{NbSyn} 87^{*} \mathrm{PEST}-n=$ 14 , all $p$ values reported are comparisons with control subjects: ${ }^{*} p<0.05,{ }^{* * *} p<0.001,{ }^{\#} p<0.05$, or as indicated on the figure. No significant findings were observed in comparison with intrabody-treated cohorts)

digestion, validating the vector-induced accumulation as authentic Lewy body-like deposition (Fig. S2). Although our model did not feature nigral neuron loss at the time of animal sacrifice, the striatal deficits that were observed serve as early hallmarks of pathology and most likely precede downstream nigral neurodegeneration. Furthermore, evidence from the VH14*PEST cohort demonstrates that intrabody treatment can directly counteract and rescue nigrostriatal terminals from the early-phase, PD-like pathology observed in the saline-treated animals.

It is unclear why, although both intrabodies successfully remediated pathogenic aggregation of a-syn, VH14*PEST showed superior histological conservation of the nigrostriatal pathway, featuring protection of striatal integration, preserved expression of striatal DAT, and trending conservation of dopaminergic nigral neurons and striatal dopamine supply. One explanation may be the increased inflammatory signaling observed in the NbSyn87*PEST-injected SN which may exacerbate a-syn-induced pathology or counteract the expected effects of reduced a-syn aggregation. ${ }^{42-44}$ The minor increases in Iba-1-labeled cells in the saline and VH14*PEST-treated cohorts may be attributed to synucleinmediated inflammation. The derivation of both nanobodies from different host species, human and camelid, provides a potential explanation in the divergent inflammatory reactions observed through microglial dynamics. The lower inflammatory response observed in the VH14*PEST group, combined with its human origin, suggests that this nanobody construct may be a more conservative therapeutic option. Stabilized inflammatory activation in the VH14*PEST treatment group also implies that viral delivery did not elicit nigral inflammation ipsilateral to injection. These data from the VH14*PEST cohort also validate that repeated injection with AAV5 virus alone does not induce stable, long-term inflammation. However, further analysis of microglial surface markers and monocyte expression is necessary to characterize any induction of active inflammation or potentiatiation of an immune response. Interestingly, NbSyn $87^{*} \mathrm{PEST}$ treatment showed a modest improvement over VH14*PEST in functional preservation in behavioral assays, in contrast to indications of ineffectual nigrostriatal dopaminergic preservation. These findings may be a result of pronounced variability in individual animal performance in both the cylinder test and the stepping test and may necessitate a lengthier lesion procedure for future experimental iterations or deeper investigation of fine motor coordination, such as gait analysis. ${ }^{45}$

An exciting attribute of intrabody therapy is the use of viral vector delivery systems as a primary modality for long-term treatment. The use of AAV vectors to deliver long-term, recombinant payloads to combat neurological disease may be ideal for prolonged, age-related neurodegeneration. ${ }^{46-48}$ Potential 

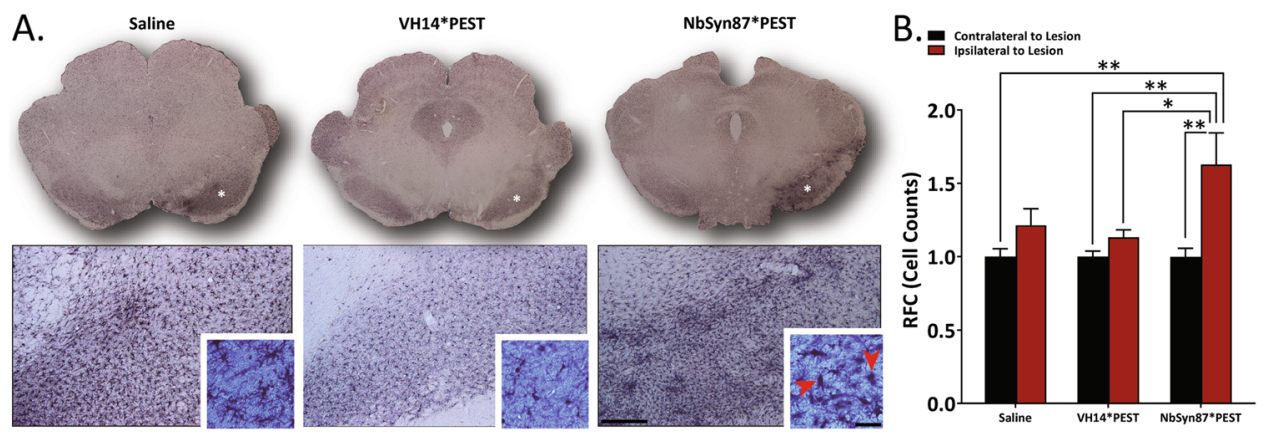

Fig. 5 NbSyn87*PEST treatment elicits inflammatory response in substantia nigra. a Immunolabeling of lba-1 in the substantia nigra reveals microglial recruitment and activation. NbSyn87*PEST treatment induces higher levels of microglial clustering to the injected substantia nigra than in VH14*PEST-treated animals. NbSyn87*PEST-treated animals also exhibit higher populations of amoeboid microglia indicating active microglial morphology (red arrow, inset) (scale bars: 100 and $25 \mu \mathrm{m}$ ). b Stereological quantification of Iba- 1 labeled cells indicates significant increases in microglial recruitment in NbSyn87*PEST, representative of a stronger inflammatory response. Data were analyzed via one-way ANOVA with Tukey's post hoc comparisons (saline $-n=6$, VH14*PEST $-n=8$, NbSyn87*PEST $-n=7$, all ${ }^{*} p<0.05,{ }^{* *} p<0.01$ )

cell replacement strategies for PD treatment may still require antia-syn therapeutic strategies, as toxic a-syn species have been shown to propagate into cell graft material. ${ }^{11}$ Intrabodies transduced into these cells pre-transplantation could protect grafted cells from host-transmitted synucleinopathy. However, a primary concern with gene therapy intrabody treatment, particularly with the deployment of proteosomal clearance mechanisms, is the effective titer and dosing paradigm required to reverse abnormal physiological concentrations of monomeric a-syn. Although early studies suggested minimal neurophysiological importance of a-syn expression, ${ }^{49}$ current data suggest that subthreshold knockdown of a-syn can induce dose-dependent neurodegeneration and functional decline in neurotransmission. $^{50,51}$ Intrabody binding to a-syn may also interrupt physiological functional capacities, primarily in membrane-binding domains critical for vesicular trafficking and SNARE (soluble $\mathrm{N}$ ethylmaleimide-sensitive factor activating protein receptor) complex arbitration. ${ }^{52,53}$ NAC region binding, as is the case for VH14*PEST, may be particularly conducive to loss-of-function asyn interference in overdosed, prolonged expression systems. Our analysis of VH14*PEST, delivered at a high titer of $1 \times 10^{13} \mathrm{vg} / \mathrm{ml}$, revealed no exacerbation of a-syn phenotypic insults. Furthermore, the lack of statistically significant decrease in humanspecific a-syn species, as denoted by $\mathrm{LB} 09^{+}$labeling (Fig. S1), suggests that the expression of VH14*PEST and NbSyn87*PEST is adequate for pathologic prevention based on the degree of a-syn overexpression in this model and maintains supra-threshold a-syn concentrations for proper function. These findings minimize concern of the potential for viral "overshoot" that may disproportionately induce excessive and detrimental clearance of a-syn, thus allowing for future studies exploring alternative delivery methods and higher-order model systems.

To date, nanobody technology has not been prominently utilized in in vivo settings. Although antibody fragments are highaffinity binders that optimize target specificity, in vitro aptamer stability has been difficult to reproduce in the cellular milieu of live animal models. The use of ER-tethered nanobodies to specifically halt transduction of protein products through the secretory pathway has shown early promise in loss-of-function targeting of toxic proteins in the context of Alzheimer's disease and tumorinduced angiogenesis. ${ }^{54}$ Through our use of the PEST motif, we were able to stabilize our constructs in a reductive cellular environment to potentially overcome this hurdle while maintaining cytosplasmic scavenging potential. Perhaps the most frequent application of in vivo nanobody utilization has been in nanobodyassisted tumor-imaging modalities for cancer-specific cellular markers. ${ }^{54-56}$ However, effective use of nanobody technology in direct target protein interference has been slow. A recent report has shown nanobody efficacy in anti-fibrillarization of circulating $\beta_{2}$-microglobulin in transgenic mice modeling genetic forms of hemodyalisis, although these nanobodies were targeting circulating protein aggregation. ${ }^{57}$ Our study is among the first to employ a gene therapy intrabody system that can specifically target amyloidogenesis of cytosolic protein within the central nervous system parenchyma.

In the context of progressing intrabody therapy into translational studies, it is important to evaluate the model system in which this set of experiments was conducted, as well as the timeline of potential therapeutic intervention. Our use of the AAVa-syn overexpression model was intended to determine the baseline efficacy of intrabody therapy on pathologic aggregation. However, clinical observation of disease is significantly delayed compared to pathogenic onset and proteopathic seeding events. A supplemental, clinically relevant experimental paradigm may also include a model utilizing pre-formed a-syn aggregates to mimic toxic cell-to-cell propagation dynamics, prion-like seed propensity, and optimize a-syn substrate clearance. ${ }^{58,59}$ Additionally, growing consideration for peripheral sources of a-syn deposition, in particular via the gut-brain axis, warrant consideration of a global therapeutic paradigm for intracellular a-syn intervention. ${ }^{60-62}$ Our study design has served as a proof-ofprinciple investigation into nanobody perturbation of modeled PD pathophysiology, primarily nigrostriatal neurotoxicity. However, clinical PD, particularly a-syn centric pathology, occurs throughout the neuraxis with prevalent manifestation of extra-motor phenotypes. Recent reports illuminating advanced transduction efficiency throughout the central nervous system and periphery via engineered AAV capsids open the door for intrabody targeting of widespread a-syn pathology potentially through intrathecal or intravenous administration, enhancing the translational potential for clinical paradigms. ${ }^{63}$ The data presented in this study, in conjunction with extracellular protein clearance mechanisms, highlight an intriguing avenue for disruption of a-syn toxicity and targeted relief of proteostatic insult in neurological disease.

\section{MATERIALS AND METHODS}

Animals

All care and husbandry of animal subjects was carried out in accordance with protocols approved by the Institutional Animal Care and Use Committee at Rush University Medical Center. Forty-eight, 8-week-old Sprague-Dawley rats (Harlan Biotechnology) were maintained under a 12hour light-dark cycle at $25^{\circ} \mathrm{C}$ and $50 \%$ humidity. Animals were fed ad libitum chow and water. All animals used in the study were acclimatized in the testing facility for 1 week prior to initiation of experimentation. 


\section{Behavioral assays}

Rodent behavioral tests were performed as previously described. ${ }^{64}$ Modified cylinder tests and stepping tests were performed 1 week prior to lesioning and 2 weeks post lesion to establish baseline performance scores. Animals were pre-sorted into treatment groups based on postlesion performance scores. Behavioral assays were conducted bi-weekly after treatment injections until the study end date after week 17. Performance for both tests was assessed by analyzing the percentage of forelimb contacts contralateral to the side of lesion.

\section{Stereotaxic surgery}

All stereotaxic surgical procedures were followed as previously described. ${ }^{65}$ Animals were anesthetized with sodium ketamine hydrochloride $(100 \mathrm{mg} / \mathrm{kg}) / x y$ lazine hydrochloride solution $(10 \mathrm{mg} / \mathrm{kg})$. All subjects were lesioned via unilateral injections of AAV5- $a$-synuclein (volume: $2 \mu \mathrm{l}$, vector titer: $1 \times 10^{12} \mathrm{vg} / \mathrm{ml}$, UNC Vector Core) into the right substantia nigra (Coordinates: $\mathrm{A} / \mathrm{P}$ at $-4.8 \mathrm{~mm}, \mathrm{M} / \mathrm{L}$ at $1.7 \mathrm{~mm}$, and $\mathrm{D} / \mathrm{V}$ at $-7.5 \mathrm{~mm}$ ) at a flow rate of $0.2 \mu \mathrm{l} / \mathrm{min}$. Animals were sorted into three treatment groups based on post-lesion behavioral performance, and a second round of surgeries were performed 3 weeks post lesion. Groups 1 and 2 received intranigral injections (volume: $2 \mu \mathrm{l}$, vector titer: $1 \times 10^{13} \mathrm{vg} /$ $\mathrm{ml}$, UNC Vector (ore) at a flow rate of $1.0 \mu \mathrm{l} / \mathrm{min}$ of AAV5-VH14*PEST and AAV5-NbSyn87*PEST, respectively. Group 3 received an injection of vehicle saline at the same injection conditions.

\section{Rodent necropsy and tissue processing}

At the study endpoint, animals were anesthetized with and perfused transcardially with $0.9 \%$ sterile phosphate-buffered saline (PBS) solution or $4 \%$ paraformaldehyde solution at $4^{\circ} \mathrm{C}$. Brains perfused with PBS were snap frozen for high-performance liquid chromatography (HPLC) processing. Brains perfused in $4 \%$ paraformaldehyde were post-fixed overnight in $4 \%$ paraformaldehyde solution and subsequently transferred to a 10,20 , and $30 \%$ sucrose gradient until the tissue sank. Brains were maintained in sucrose solution until they were cut on a freezing-stage sliding microtome into $40 \mu \mathrm{m}$ coronal sections. Sections were stored in cryopreservative solution until immunohistochemical processing.

\section{High-performance liquid chromatography}

All HPLC protocols were followed as previously described. ${ }^{66}$ Striata from eight animals in each cohort were extracted, processed, and analyzed for concentrations of dopamine. Tissue was sonicated in $0.2 \mathrm{M}$ perchloric acid with isoproterenol. Centrifuged supernatant was injected in a Eicompak SC-3ODS column (JM Science, Inc.) and analyzed as per the manufacturer's protocol.

Immunohistochemistry, Proteinase $\mathrm{K}$, and Thioflavin-S staining All immunohistochemistry protocols were followed as previously described. ${ }^{65}$ Free-floating sections $(40 \mu \mathrm{m})$ of coronal brain slices were stored in cryoprotectant and quenched with sodium periodate prior to staining. Sections for immunohistochemistry were incubated with the primary antibodies listed in Table S1. Sections were subsequently incubated with the respective biotinylated secondary antibodies (Table S1) and labeled with a standard ABC HRP Biotin/Avidin Complex Kit (Vector Laboratories). Staining was developed with $D A B$ and sections were mounted for imaging and analysis. Proteinase $\mathrm{K}$ digestion protocol was followed as previously described. ${ }^{65}$ Briefly, sections were mounted and dried on gelatin-coated slides. Tissue was rehydrated and incubated with $10 \mu \mathrm{l} / \mathrm{ml}$ of Proteinase $\mathrm{K}$ (Invitrogen) dissolved in TBST for $30 \mathrm{~min}$ at $55^{\circ} \mathrm{C}$. Sections were subsequently fixed with $4 \%$ paraformaldehyde for $10 \mathrm{~min}$ and immunostained for a-syn as previously described. Preceding Thioflavin-S staining, tissue was pre-processed for phosphorylated S129 a-syn immunofluorescence using adapted staining protocol previously described. Tissue was mounted and dried on gelatin-coated slides at room temperature. Sections were defatted in chloroform for $2 \mathrm{~h}$ and hydrated through graded alcohols. Tissue was incubated in $0.1 \%$ Thioflavine-S solution (Sigma) for $10 \mathrm{~min}$ prior to imaging. All immunofluorescent images were taken on an Olympus confocal laser scanning microscope with the Fluoview software.

\section{Densitometry}

Optical density measurements were taken using Scion Image (v1.63, Meyer Instruments). Samples were imaged on an Olympus BH-2 microscope (Olympus) and corresponding regions imaged were aligned in a reading frame prior to recording. Densitometric measurements from a minimum of two sections in a half-series (every 12th section) of each subject were recorded and averaged for quantitative measurements. Data from variable regions were normalized with in-subject, uninjected control hemispheres.

\section{Stereology}

Cell counts were estimated by unbiased stereological counting methods using the optical fractionator probe in Stereolnvestigator (v10.40, MBF Biosciences). ${ }^{67}$ All raters were blinded to subject ID prior to analysis. Sections were viewed on an Olympus BX51 microscope (Olympus) with specific brain region contours detected and traced at $\times 4$ objective and data recordings conducted with $\times 60$ magnification. Serial sections of biological replicates were recorded using every 12 sections of $40 \mu \mathrm{m}$ coronal slices. Stereological assessments of $\mathrm{TH}^{+}$-labeled cells were used to establish degree of lesion affecting dopaminergic neurons in the substantia nigra. To assess quantitatively the level of inflammation at the site of a given lesion, Iba- $1^{+}$-labeled cells were also counted in the substantia nigra.

\section{Statistical analysis}

All data are presented as mean $\pm \mathrm{SEM}$. All statistical analyses were performed using the GraphPad Prism (version 7.03). Statistical differences were determined by one-way or two-way analysis of variance, with corrections for repeated measures as needed. Specific statistical analyses and significant findings are noted in figure legends.

\section{Data availability}

All relevant data and analyses from this study are available from the corresponding author upon request.

\section{ACKNOWLEDGEMENTS}

This study was supported by a Center Grant from the Parkinson's Disease Foundation (PDF-02, J.H.K., D.C., M.B., A.M., and D.B.) and a Rapid Response Innovation Award from the Michael J. Fox Foundation (J.H.K., D.C., M.B., A.M., and D.B.). This work was additionally supported in part by the Cambridge Centre for Misfolding Diseases (C.M. D. and E.D.G.). We would like to thank the UNC Vector Core for the viral constructs utilized in the study.

\section{AUTHOR CONTRIBUTIONS}

Conceived and designed the study: J.H.K., A.M., and D.B. Contributed experimental materials: E.D.G. and C.M.D. Conducted the experiments: D.C. and M.B. Analyzed the data, prepared the figures, and wrote the manuscript: D.C. Validated and approved data and analysis: J.H.K. and A.M. Edited, modified, and approved the manuscript: J.H. K, A.M., D.B., E.D.G., C.M.D., and D.C.

\section{ADDITIONAL INFORMATION}

Supplementary information accompanies the paper on the npj Parkinson's Disease website (https://doi.org/10.1038/s41531-018-0062-4).

Competing interests: The authors declare no competing interests.

Publisher's note: Springer Nature remains neutral with regard to jurisdictional claims in published maps and institutional affiliations.

\section{REFERENCES}

1. Kalia, L. V. \& Lang, A. E. Parkinson's disease. Lancet 386, 896-912 (2015).

2. Obeso, J. A. et al. Past, present, and future of Parkinson's disease: a special essay on the 200th Anniversary of the Shaking Palsy. Mov. Disord. 32, 1264-1310 (2017).

3. Spillantini, M. G., Crowther, R. A., Jakes, R., Hasegawa, M. \& Goedert, M. Alphasynuclein in filamentous inclusions of Lewy bodies from Parkinson's disease and dementia with lewy bodies. Proc. Natl. Acad. Sci. USA 95, 6469-6473 (1998). 
4. Ingelsson, M. Alpha-synuclein oligomers-neurotoxic molecules in Parkinson's disease and other Lewy body disorder. Front. Neurosci. 10, 408 (2016).

5. Schildknecht, S. et al. Oxidative and nitrative alpha-synuclein modifications and proteostatic stress: implications for disease mechanisms and interventions in synucleinopathies. J. Neurochem. 125, 491-511 (2013).

6. Xiang, W. et al. Oxidative stress-induced posttranslational modifications of alphasynuclein: specific modification of alpha-synuclein by 4-hydroxy-2-nonenal increases dopaminergic toxicity. Mol. Cell Neurosci. 54, 71-83 (2013).

7. Kanda, S., Bishop, J. F., Eglitis, M. A., Yang, Y. \& Mouradian, M. M. Enhanced vulnerability to oxidative stress by alpha-synuclein mutations and C-terminal truncation. Neuroscience 97, 279-284 (2000).

8. Pukass, K., Goldbaum, O. \& Richter-Landsberg, C. Mitochondrial impairment and oxidative stress compromise autophagosomal degradation of alpha-synuclein in oligodendroglial cells. J. Neurochem. 135, 194-205 (2015).

9. Shtilerman, M. D., Ding, T. T. \& Lansbury, P. T. Jr. Molecular crowding accelerates fibrillization of alpha-synuclein: could an increase in the cytoplasmic protein concentration induce Parkinson's disease? Biochemistry 41, 3855-3860 (2002).

10. Dehay, B., Vila, M., Bezard, E., Brundin, P. \& Kordower, J. H. Alpha-synuclein propagation: New insights from animal models. Mov. Disord. 31, 161-168 (2016).

11. Kordower, J. H. \& Brundin, P. Propagation of host disease to grafted neurons: accumulating evidence. Exp. Neurol. 220, 224-225 (2009)

12. Kordower, J. H. \& Brundin, P. Mechanisms for cell-to-cell propagation no longer lag behind. Mov. Disord. 31, 1798-1799 (2016).

13. El-Agnaf, O. et al. Differential effects of immunotherapy with antibodies targeting alpha-synuclein oligomers and fibrils in a transgenic model of synucleinopathy. Neurobiol. Dis. 104, 85-96 (2017).

14. Spencer, B. et al. Anti-alpha-synuclein immunotherapy reduces alpha-synuclein propagation in the axon and degeneration in a combined viral vector and transgenic model of synucleinopathy. Acta Neuropathol. Commun. 5, 7 (2017).

15. Tran, H. T. et al. Alpha-synuclein immunotherapy blocks uptake and templated propagation of misfolded alpha-synuclein and neurodegeneration. Cell reports 7 , 2054-2065, (2014).

16. Valera, E. et al. Combination of alpha-synuclein immunotherapy with antiinflammatory treatment in a transgenic mouse model of multiple system atrophy. Acta Neuropathol. Commun. 5, 2 (2017).

17. Messer, A. \& Joshi, S. N. Intrabodies as neuroprotective therapeutics. Neurother. J. Am. Soc. Exp. Neurother. 10, 447-458 (2013).

18. Bhatt, M. A., Messer, A. \& Kordower, J. H. Can intrabodies serve as neuroprotective therapies for Parkinson's disease? Beginning thoughts. J. Parkinson's Dis. 3, 581-591 (2013).

19. De Genst, E., Messer, A. \& Dobson, C. M. Antibodies and protein misfolding: from structural research tools to therapeutic strategies. Biochim. Biophys. Acta 1844, 1907-1919 (2014).

20. Lynch, S. M., Zhou, C. \& Messer, A. An scFv intrabody against the nonamyloid component of alpha-synuclein reduces intracellular aggregation and toxicity. $J$. Mol. Biol. 377, 136-147 (2008).

21. El-Turk, F. et al. Structural effects of two camelid nanobodies directed to distinct C-terminal epitopes on alpha-synuclein. Biochemistry 55, 3116-3122 (2016).

22. Butler, D. C. et al. Bifunctional anti-non-amyloid component alpha-synuclein nanobodies are protective in situ. PLOS ONE 11, e0165964 (2016).

23. Guilliams, T. et al. Nanobodies raised against monomeric alpha-synuclein distinguish between fibrils at different maturation stages. J. Mol. Biol. 425 2397-2411 (2013).

24. Eliezer, D. The mysterious C-terminal tail of alpha-synuclein: nanobody's guess. J. Mol. Biol. 425, 2393-2396 (2013).

25. Iljina, M. et al. Nanobodies raised against monomeric a-synuclein inhibit fibril formation and destabilize toxic oligomeric species. BMC Biol. 15, 57 (2017).

26. Joshi, S. N., Butler, D. C. \& Messer, A. Fusion to a highly charged proteasomal retargeting sequence increases soluble cytoplasmic expression and efficacy of diverse anti-synuclein intrabodies. mAbs 4, 686-693 (2012).

27. Butler, D. C. \& Messer, A. Bifunctional anti-huntingtin proteasome-directed intrabodies mediate efficient degradation of mutant huntingtin exon 1 protein fragments. PLoS ONE 6, e29199 (2011).

28. Decressac, M., Mattsson, B., Lundblad, M., Weikop, P. \& Bjorklund, A. Progressive neurodegenerative and behavioural changes induced by AAV-mediated overexpression of alpha-synuclein in midbrain dopamine neurons. Neurobiol. Dis. 45, 939-953 (2012).

29. Games, D. et al. Reducing C-terminal-truncated alpha-synuclein by immunotherapy attenuates neurodegeneration and propagation in Parkinson's disease-like models. J. Neurosci. 34, 9441-9454 (2014).

30. Wong, Y. C. \& Krainc, D. Alpha-synuclein toxicity in neurodegeneration: mechanism and therapeutic strategies. Nat. Med. 23, 1-13 (2017).

31. Menges, S. et al. Alpha-synuclein prevents the formation of spherical mitochondria and apoptosis under oxidative stress. Sci. Rep. 7, 42942 (2017).
32. Pukass, K. \& Richter-Landsberg, C. Oxidative stress promotes uptake, accumulation, and oligomerization of extracellular alpha-synuclein in oligodendrocytes. J. Mol. Neurosci. 52, 339-352 (2014).

33. Burbulla, L. F. et al. Dopamine oxidation mediates mitochondrial and lysosomal dysfunction in Parkinson's disease. Science 357, 1255-1261 (2017).

34. Jiang, T. F. \& Chen, S. D. Dysfunction of two lysosome degradation pathways of alpha-synuclein in Parkinson's disease: potential therapeutic targets? Neurosci. Bull. 28, 649-657 (2012).

35. Wang, M. X. et al. TNF compromises lysosome acidification and reduces alphasynuclein degradation via autophagy in dopaminergic cells. Exp. Neurol. 271 112-121 (2015).

36. Lim, J. \& Yue, Z. Neuronal aggregates: formation, clearance, and spreading. Dev. Cell 32, 491-501 (2015)

37. Burke, R. E. \& O'Malley, K. Axon degeneration in Parkinson's disease. Exp. Neurol. 246, 72-83 (2013).

38. Kordower, J. H. et al. Disease duration and the integrity of the nigrostriatal system in Parkinson's disease. Brain 136, 2419-2431 (2013).

39. Chu, Y., Buchman, A. S., Olanow, C. W. \& Kordower, J. H. Do subjects with minimal motor features have prodromal Parkinson disease? Ann. Neurol. 83, 562-574 (2018).

40. Kramer, M. L. \& Schulz-Schaeffer, W. J. Presynaptic alpha-synuclein aggregates, not Lewy bodies, cause neurodegeneration in dementia with Lewy bodies. J. Neurosci. 27, 1405-1410 (2007).

41. Kordower, J. H. et al. Robust graft survival and normalized dopaminergic innervation do not obligate recovery in a Parkinson disease patient. Ann. Neurol. 81 46-57 (2017).

42. Hunter, R. L. et al. Inflammation induces mitochondrial dysfunction and dopaminergic neurodegeneration in the nigrostriatal system. J. Neurochem. 100, 1375-1386 (2007).

43. Kones, R. Parkinson's disease: mitochondrial molecular pathology, inflammation, statins, and therapeutic neuroprotective nutrition. Nutr. Clin. Pract. 25, 371-389 (2010).

44. Teismann, P. \& Schulz, J. B. Cellular pathology of Parkinson's disease: astrocytes, microglia and inflammation. Cell Tissue Res. 318, 149-161 (2004).

45. Asakawa, T. et al. Human behavioral assessments in current research of Parkinson's disease. Neurosci. Biobehav. Rev. 68, 741-772 (2016).

46. Choong, C. J., Baba, K. \& Mochizuki, H. Gene therapy for neurological disorders. Exp. Opin. Biol. Ther. 16, 143-159 (2016).

47. Kamran, N. et al. Gene therapy for the treatment of neurological disorders: central nervous system neoplasms. Methods Mol. Biol. 1382, 467-482 (2016).

48. Sahin, M. Gene therapy for childhood neurological disease. Pediatr. Neurol. 51 595-596 (2014).

49. Abeliovich, A. et al. Mice lacking alpha-synuclein display functional deficits in the nigrostriatal dopamine system. Neuron 25, 239-252 (2000).

50. Collier, T. J., Redmond, D. E. Jr, Steece-Collier, K., Lipton, J. W. \& Manfredsson, F. P. Is alpha-synuclein loss-of-function a contributor to parkinsonian pathology? Evidence from non-human primates. Front. Neurosci. 10, 12 (2016).

51. Gorbatyuk, O. S. et al. In vivo RNAi-mediated alpha-synuclein silencing induces nigrostriatal degeneration. Mol. Ther. 18, 1450-1457 (2010).

52. Fusco, G. et al. Structural basis of synaptic vesicle assembly promoted by alphasynuclein. Nat. Commun. 7, 12563 (2016).

53. Fusco, G. et al. Structural basis of membrane disruption and cellular toxicity by alpha-synuclein oligomers. Science 358, 1440-1443 (2017).

54. Marschall, A. L., Dubel, S. \& Boldicke, T. Specific in vivo knockdown of protein function by intrabodies. mAbs 7, 1010-1035 (2015).

55. Fumey, W. et al. Nanobodies effectively modulate the enzymatic activity of CD38 and allow specific imaging of CD38(+) tumors in mouse models in vivo. Sci. Rep. 7, 14289 (2017).

56. Bannas, P. et al. Molecular imaging of tumors with nanobodies and antibodies: timing and dosage are crucial factors for improved in vivo detection. Contrast Media Mol. Imag. 10, 367-378 (2015).

57. Raimondi, S. et al. A specific nanobody prevents amyloidogenesis of D76N beta2microglobulin in vitro and modifies its tissue distribution in vivo. Sci. Rep. 7 46711 (2017).

58. Thakur, P. et al. Modeling Parkinson's disease pathology by combination of fibril seeds and alpha-synuclein overexpression in the rat brain. Proc. Natl. Acad. Sci. USA 114, E8284-E8293 (2017).

59. Nouraei, N. et al. Critical appraisal of pathology transmission in the alphasynuclein fibril model of Lewy body disorders. Exp. Neurol. 299, 172-196 (2018).

60. Grathwohl, S. A., Steiner, J. A., Britschgi, M. \& Brundin, P. Mind the gut: secretion of alpha-synuclein by enteric neurons. J. Neurochem. 125, 487-490 (2013).

61. Olanow, C. W., Wakeman, D. R. \& Kordower, J. H. Peripheral alpha-synuclein and Parkinson's disease. Mov. Disord. 29, 963-966 (2014).

62. Shannon, K. M. et al. Alpha-synuclein in colonic submucosa in early untreated Parkinson's disease. Mov. Disord. 27, 709-715 (2012). 
63. Chan, K. Y. et al. Engineered AAVs for efficient noninvasive gene delivery to the central and peripheral nervous systems. Nat. Neurosci. 20, 1172-1179 (2017).

64. Cederfjall, E. et al. Continuous DOPA synthesis from a single AAV: dosing and efficacy in models of Parkinson's disease. Sci. Rep. 3, 2157 (2013).

65. Mandel, R. J. et al. Novel oligodendroglial alpha synuclein viral vector models of multiple system atrophy: studies in rodents and nonhuman primates. Acta Neuropathol. Commun. 5, 47 (2017).

66. Chandra, G., Rangasamy, S. B., Roy, A., Kordower, J. H. \& Pahan, K. Neutralization of RANTES and Eotaxin prevents the loss of dopaminergic neurons in a mouse model of Parkinson disease. J. Biol. Chem. 291, 15267-15281 (2016).

67. West, M. J. \& Gundersen, H. J. Unbiased stereological estimation of the number of neurons in the human hippocampus. J. Comp. Neurol. 296, 1-22 (1990).
Open Access This article is licensed under a Creative Commons Attribution 4.0 International License, which permits use, sharing, adaptation, distribution and reproduction in any medium or format, as long as you give appropriate credit to the original author(s) and the source, provide a link to the Creative Commons license, and indicate if changes were made. The images or other third party material in this article are included in the article's Creative Commons license, unless indicated otherwise in a credit line to the material. If material is not included in the article's Creative Commons license and your intended use is not permitted by statutory regulation or exceeds the permitted use, you will need to obtain permission directly from the copyright holder. To view a copy of this license, visit http://creativecommons. org/licenses/by/4.0/.

(c) The Author(s) 2018 